\title{
Extradural ganglioneuroma with T1-T2 involvement mimicking spondylodiscitis: a case report and a review of the literature
}

Mehdi Mogharrabi', Ramin Azarhoush², Hamid Javadi ${ }^{3}$, Elahe Pirayesh ${ }^{4}$, Majid Assadi ${ }^{5}$

'Department of Nuclear Medicine, ${ }^{\text {th }}$ Azar Hospital, Golestan University of Medical Sciences (GUOMS), Gorgan, Iran

2Department of Pathology, Golestan University of Medical Sciences (GUOMS), Gorgan, Iran

${ }^{3}$ Golestan Research Center of Gastroenterology and Hepatology (GRCGH), Golestan University of Medical Sciences (GUOMS), Gorgan, Iran

${ }^{4}$ Department of Nuclear Medicine, Shohada e Tajrish Medical Center, Shahid Beheshti University of Medical Sciences, Tehran, Iran

${ }^{5}$ The Persian Gulf Nuclear Medicine Research Center, Bushehr University of Medical Sciences, Bushehr, Iran

[Received 1 II 2014; Accepted 18 VIII 2016]

\begin{abstract}
Ganglioneuroma (GN) is a rare benign neural tumor, usually derived from the ganglia of the sympathetic system. This report describes a 36-year-old man who presented with back pain and local tenderness that closely mimicked the clinical and imaging findings of spondylodiskitis. However, histologic examination made the diagnosis of GN. To our knowledge, this is the first report presenting the pattern of a GN as a differential diagnosis of spondylodiskitis.
\end{abstract}

KEY words: neural crest tumor, spinal cord neoplasms, ganglioneuroma, radionuclide imaging

Nucl Med Rev 2016; 19, Suppl. B: B3-B4

\section{Background}

Ganglioneuroma (GN) is a rare benign neural tumor, usually derived from the ganglia of the sympathetic system [1]. The most common anatomical sites are the mediastinum, retroperitoneum, adrenal gland and also head and neck [1]. This report describes a 36-year-old man presenting with back pain and local tenderness, which closely mimicked the clinical and imaging findings of spondylodiskitis. However, histologic examination demonstrated the diagnosis of GN.

\section{Case report}

A 36-year-old man presented with a history of progressive back pain for three months. His past medical, surgical and family history were unremarkable. He denied any other symptoms such as fever, night sweets, cough, breathing difficulty and weight loss. In physical examination, except local tenderness over upper thoracic vertebrae, no other abnormal finding was found. Neurologic examination was also normal. Laboratory data revealed hemoglobin $10 \mathrm{~g} / \mathrm{dL}$, hematocrit 27.8\%, mean corpuscular volume (MCV) $75.54 \mu \mathrm{m}^{3}$, erythrocyte sedimentation rate (ESR) $92 \mathrm{~mm} / \mathrm{h}$ and CRP 2+. The

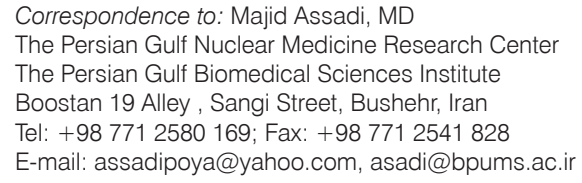

patient subsequently underwent magnetic resonance imaging (MRI) of thorax, which revealed signal changes of T1, T2 bodies, erosion of the endplates with invasion into the intervertebral disc space and a soft tissue mass, bulged anteriorly. The canal was preserved. For three phasic bone scans, immediately following injection of $20 \mathrm{mCi}$ 99mTcMDP, bone scan was obtained in perfusion and blood pool phases from thoracic vertebrae. Delayed whole-body bone scan with spot views from that region was also performed. The finding was regional hyperemia and increased radiotracer uptake of T1-T2 vertebrae (Figure 1). Remainder of the skeleton was unremarkable.

Altogether, the clinical history, laboratory data and imaging findings were suggestive of spondylodiscitis, most probably spinal tuberculosis [2], which is not an uncommon disease in our country $[3,4]$. The patient underwent open biopsy which histologic analysis of specimen derived from the spinal mass demonstrated mature ganglion cells, with abundant cytoplasm, large nuclei and prominent nucleoli, distributed within an abundant stroma, compatible with ganglioneuroma (Figure 2).

\section{Discussion}

Sympathetic trunks and their associated ganglia, located on the side of vertebral bodies, can be the origin of sympathetic ganglion tumors. These include a spectrum from aggressively malignant neuroblastoma (NB), to malignant ganglioneuroblastoma (GNB) and to purely benign GN. Gangliocytoma is a rare and benign tumor arising from ganglia-type cells, which are groups of nerve cells. This tumor arises from ganglia. When neuronal elements make up the majority 
A

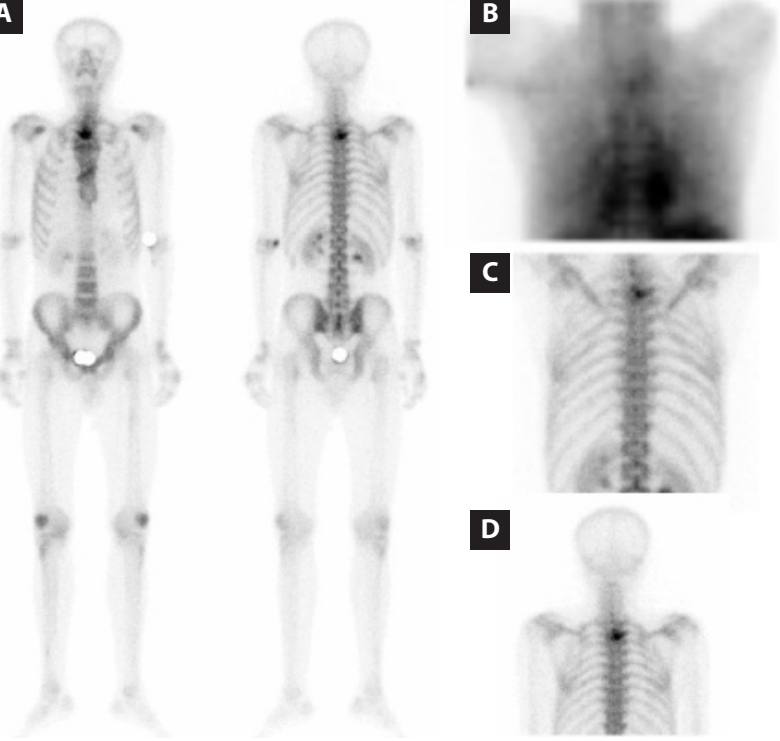

Figure 1A-D. Whole body and three phasic bone scintigraphy using $99 \mathrm{~m}$ technetium methylene diphosphonate demonstrated regional hyperemia and increased radiotracer uptake of T1-T2 vertebrae. Remainder of the skeleton was unremarkable

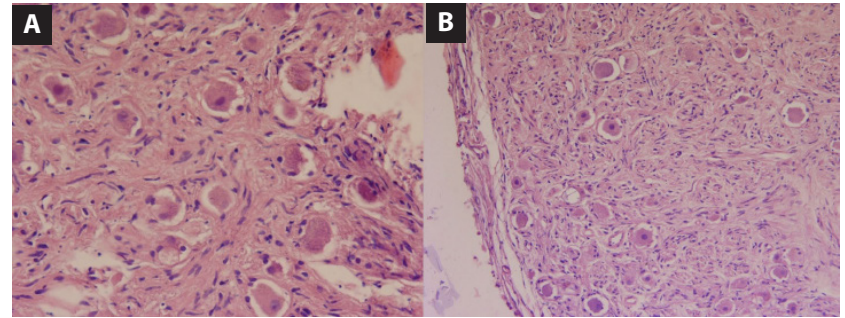

Figure 2A, B. Histologic analysis of specimen derived from the spinal mass in microscopic view $(\mathrm{H \& E}, \times 400)$ demonstrated mature ganglion cells, with abundant cytoplasm, large nuclei and prominent nucleoli, distributed within an abundant stroma, compatible with ganglioneuroma

of the mass, the tumor is referred to as a ganglioneuroma. In contrast, gangliocytomas are composed of mature ganglion cells and therefore have no potential for malignant change. Gangliocytoma is a surgically curable disease and surgical resection performs in symptomatic patients. Overall, prognosis is good and recurrence rate is about 25 percent.

Peripheral GN is a well differentiated, benign and slowly growing tumor. It can arise anywhere from the base of the skull to the pelvis, but its most common location is the posterior mediastinum (41.5\%), followed by retroperitoneum (37.5\%), adrenal gland (21\%) and neck $(8 \%)[1,5]$. Spinal GN usually involves the paraspinal region and can grow into the spinal canal through the neural foramina [6]. Depending on the location of the tumor, spinal GN may cause various symptoms, including scoliosis, peripheral radiculopathy, paraparesis or gait disturbance [6, 7]. Although, GN is a benign well- encapsulated tumor and typically does not invade adjacent structures, it has been reported that it can attach to the vertebral bodies [8], or involve the spinal column [6, 9-11]. However, in our patient a unique pattern of bone involvement was noted, mimicking spondylodiscitis. In addition, in most of reported cases, scintigraphic appearance of bone involvement has not been mentioned. In our knowledge, this is the first report presenting the pattern of a GN as a differential diagnosis of spondylodiskitis.

The diagnostic value of metaiodobenzylguanidine (MIBG) scintigraphy in neural crest / neuroblast-derived tumors such as neuroblastoma and pheochromocytoma is established [12, 13].

It should be mentioned that main differential diagnoses of such pattern in the thoracic spine on bone scan are inflammatory process and tumoral involvement, for which radiolabeled WBC's and gallium scanning in addition to radiological modalities, especially $\mathrm{MRI}$, are helpful to reach the diagnosis of inflammation [14].

\section{References}

1. Mounasamy V, Thacker M, Humble S et al. Ganglioneuromas of the sacrum - a report of two cases with radiologic-pathologic correlation. Skeletal Radiol 2006; 35: 117-121.

2. Trecarichi EM, Meco ED, Mazzotta V, Fantoni M, Tuberculous spondylodiscitis: epidemiology, clinical features, treatment, and outcome. Eur Rev Med Pharmacol Sci 2012; 16: 58-72.

3. Alavi SM, Sharifi M. Tuberculous spondylitis: Risk factors and clinical/paraclinical aspects in the south west of Iran. J Infect Public Health 2010; 3: 196-200.

4. Assadi M, Nabipour I, Eftekhari M et al. Diagnostic role of whole body bone scintigraphy in atypical skeletal tuberculosis resembling multiple metastases: a case report. J Med Case Rep 2009; 3: 141.

5. Nakazono T, White CS, Yamasaki F et al. MRI findings of mediastinal neurogenic tumors. AJR Am J Roentgenol 2011; 197: W643-W652.

6. Pang BC, Tchoyoson Lim CC, Tan KK. Giant spinal ganglioneuroma. J Clin Neurosci 2005: 12: 967-972

7. Cote P, Cassidy JD, Dzus A, Yong-Hing K. Ganglioneuroma of the thoracic spine presenting as adolescent idiopathic scoliosis: a case report. J Spinal Disord 1994; 7: 528-532

8. Forsythe A, Volpe J, Muller R. Posterior mediastinal ganglioneuroma. Radiographics 2004; 24: 594-597.

9. Zhang J, Li J, Shrestha R, Jiang S. Giant cervicothoracic ganglioneuroma. Neurol India 2011; 59: 465-466.

10. Chen M, Zhang J, Huang S, You C. Complicated lumbar ganglioneuroma. Neurol India 2012; 60: 539-540

11. Chen Y-H, Tsai Y-C, Yen C-H, Lai C-W, Shiau Y-C. Bone invasion of retroperitoneal ganglioneuroma detected by bone scan but not by computed tomography: a case report. Ann Nucl Med Sci 2008; 21: 121-126.

12. Leung A, Shapiro B, Hattner $R$ et al. Specificity of radioiodinated MIBG for neural crest tumors in childhood. J Nucl Med 1997: 38: 1352-1357.

13. Sasajima T, Kinouchi H, Tomura N, Watarai J, Mizoi K. High uptake of 123I-metaiodobenzylguanidine related to olfactory neuroblastoma revealed by single-photon emission CT. AJNR Am J Neuroradiol 2000; 21: 717-720.

14. Kolindou A, Liu Y, Ozker $\mathrm{K}$ et al. In-111 WBC imaging of osteomyelitis in patients with underlying bone scan abnormalities. Clin Nucl Med 1996; 21: 183-191. 